\title{
3D Modeling of X-Ray Images: A Review
}

\author{
Baishali Goswami \\ Department of Computer Science and Engineering \\ Sikkim Manipal Institute of Technology, \\ Sikkim, India
}

\begin{abstract}
Accurate geometrical reconstruction of human bones into three-dimensional(3D) view is currently required for clinical studies such as enabling the radiologists to well analyze the fractures or infections in the bones, evidence of arthritis, presence of dental decays, lung infections etc. CT-scan is commonly used to obtain accurate reconstruction of the human body. However, this method is quite relucent for the patients as it demands a large number of image data sets, typically, more than 100s of images for a single bone to reconstruct. Analysis using MRI are also meant especially to investigate the anatomy and physiology of the body in both health and disease. However, although quite accurate, CTscan is not an appropriate $3 \mathrm{D}$ reconstruction method because of the high irradiating dose, high price and large input data volume. Thus, a 3D model reconstructed from 2D X-ray images can be a useful alternative. The generation of the 3D model is termed as 3D reconstruction from 2D X-Ray images. The reconstruction of the X-ray images can be achieved from both single and multiple X-Ray images. Many researches have been carried out in this field and the reconstruction has been carried out with varying accuracy. This paper presents a review of the existing methods for accurate 3D reconstruction from bi-planar X-rays.
\end{abstract}

\section{General Terms}

3D Reconstruction, X-Ray images.

\section{Keywords}

3D Reconstruction; Hough transform; Laplacian deformation; Contour matching; Medical modeling software.

\section{INTRODUCTION}

Three-dimensional model reconstruction of bone anatomy from medical images has been proven to be very useful for preoperative planning and computer-aided surgeries. The conventional methods of reconstruction of 3D model from CT or MRI images mostly deal with image processing and visualization techniques, and the $3 \mathrm{D}$ information is already present in the images. These methods, however, have drawbacks such as expensive imaging techniques, high volume of data, more time to generate 3D model and lesser availability. In addition, CT scanning also involves drawbacks of high radiation. Hence, 3D modeling from two $\mathrm{X}$-ray images using some prior information about the bone shape and shape variation has become a topic of research interest. The methods of 3D reconstruction from X-ray images can be classified according to the information used for reconstruction. At one extreme, there are methods in which only the X-ray images are used as the inputs. In these methods, the contours extracted from the calibrated X-ray images are back-projected to approximate the cross section of the bone shape. On the other extreme, there are methods which use a mathematical model containing information of the bone shape as well as its variation. This is referred to as statistical shape model (SSM), created by statistical analysis of a collection of bone shapes. The SSM has information about the average shape geometry and shape variations. The

\author{
Santanu Kr. Misra \\ Department of Computer Science and Engineering \\ Sikkim Manipal Institute of Technology, \\ Sikkim, India
}

average shape can be deformed by applying a linear combination of principal modes of shape variations contained in the SSM. The deformation is performed until the projection of the deformed shape matches the information from X-ray images. Methods based on inputs from X-ray images alone do not give good accuracy since the images have limited information. Methods based on SSM have shown more accurate results but they require a large database. The database can be classified according to age, gender, and ethnicity. The template-based methods using FFD for template reconfiguration have been considered to be a viable option for developing software applications for 3D reconstruction. This paper presents a review of the existing methods for accurate $3 \mathrm{D}$ reconstruction from bi-planar X-ray images.

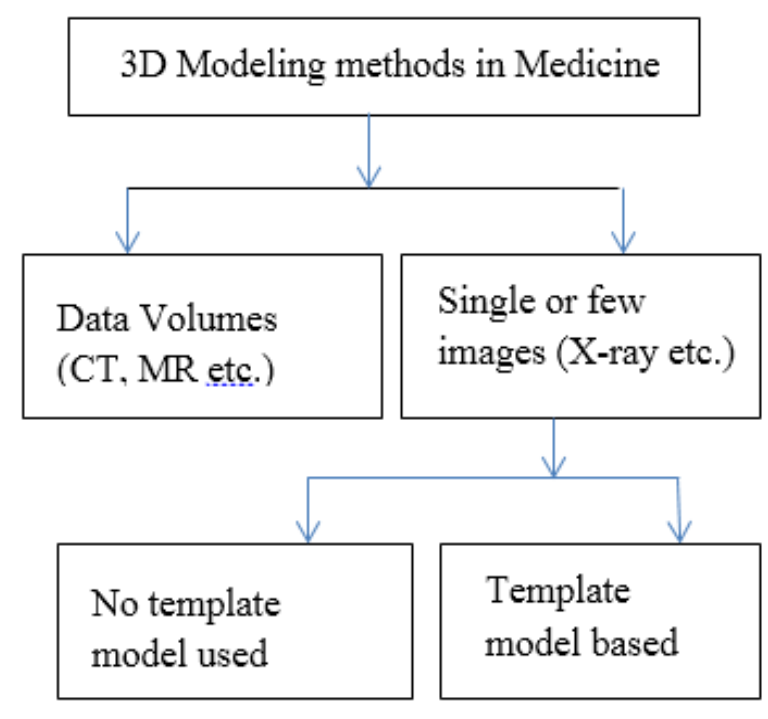

Fig. 1. Classification of 3D modeling methods of medical images.

\section{RELATED WORKS}

Cresson et.al [1] in their paper proposed a non-rigid registration method to estimate the 3D personalized shape of bone models using two bi-planar X-ray images based on asrigid-as-possible deformation approach following a moving least-squares optimization method. The interactive deformation method of the technique provides the user with the ability of improving the 3D reconstruction procedure readily and in a simplified manner. In this paper, they adapt the deformation algorithm developed by Schaefer et al. to align the template surface model with the $2 \mathrm{D}$ bone contours extracted from x-ray images of the patient.

Rizquie et.al [2] in their paper described the first step of the $3 \mathrm{D}$ reconstruction using $2 \mathrm{D} \mathrm{X}$-ray images as subtracting the ribcage from the images, and then applying ribcage reconstruction. The next step followed is to segment the lung area, then reconstruct it by creating a mesh that encompasses 
the inside of the reconstructed ribcage, and then carves out the portion of that mesh, which corresponds to the lungs by extruding the segmented lungs up through it. Finally, the surface of chest area can be applied from the segmentation of the edge of the images.

Baka et. Al [3] in their paper presented a method to reconstruct the anatomy of the bone using two or more calibrated X-ray images using a statistical shape model and an objective function based on 3D distance. It uses the edges produced by the canny operator in the projected images. The proposed method performs the process of reconstruction automatically with the exception of the initial pose estimate.

Branchaud et.al [4] proposed a method that is based on combining the partial information from two images taken from a subject while generating a statistical 3D spine model from a directory of patients in an efficient manner. The algorithm proceeds with two simultaneous iterating processes. The first process produces a personalized vertebra model adopting a $2 \mathrm{D} / 3 \mathrm{D}$ registration process. Whereas, the latter accounts for the position and the shape of the other remaining vertebraes from the estimate currently produced regarding the process of registration adopting a statistical 3D model.

Ehlke et.al [5] proposed a novel GPU-based approach that outputs X-ray projections virtually for tetrahedral meshes that are deformed. The shape and the internal density distribution of a distinct anatomical structure are represented using these meshes. These are further derived using statistical shape and intensity models (SSIMs). They applied their method to make an improvement to the geometric reconstruction of 3D anatomy using 2D X-ray images. In regard to this, variations are made into the shape and density functions of a tetrahedral mesh and generation of virtual Xray projections is carried out within an optimized function so that a maximum similarity is achieved between the computed virtual $\mathrm{X}$-ray and the respective anatomy.

Galibarov et. Al [6] presented a method that creates 3D surface models in an automated manner from bi-planar radiographs. The method consists of (i) extraction of contour from the X-Ray image using an Active Contour (Snake) algorithm, (ii) selection of the closest matching 3D model from a library of generic models, and (iii) improving the correlation with the extracted contour by warping the selected generic model. One benefit of the technique proposed in this study is that it is highly automated and able to generate surface models rapidly.

Gamage et.al [7] in their paper, proposed a technique in which the 2D X-ray images are initially processed to extract the edge points that potentially form the femur boundary. The identified point correspondences are then interpolated to create a 2D planar translational field in both the anterior and lateral viewpoints. This translational field will identify the deformations required by the $3 \mathrm{D}$ anatomical model in the equivalent viewpoint. Finally a full 3D translational field is created through a thin plate spline based interpolation and the 3D generic anatomical data deformed accordingly. The proposed shape reconstruction framework utilizes a generic 3D surface model to provide the necessary cross-sectional topology constraints.

Oliver [8] in his work described and analyzed common rendering techniques and their attributes. He designed and implemented a volume renderer in Java using the Java API Java3D. The author implemented VolVi3D, a plug-in for the
Java-based image processing tool ImageJ. VolVi3D works completely with the original data provided by ImageJ. The user can continue to modify his or her image stack in ImageJ and can update the displayed 3D model in VolVi3D every times he or she wants.

Lamecker et.al [9] presents a method to reconstruct 3D models from few digital X-ray images based on the concept of 3D-statistical shape models. This method relies extensively on an algorithm which calculates an optimization function for calculating a measure that defines the similarity factor for assessment of the distance between shape model projection and the given X-ray images. Their idea is the measurement of the difference existing between the object silhouettes present in the respective projections based on theoretical and experimental observations. The shape model considered in this research is produced from a collection of individual training shapes (triangulated surfaces). The main objective behind this work lies in the correct identification of corresponding points anatomically from each training surface.
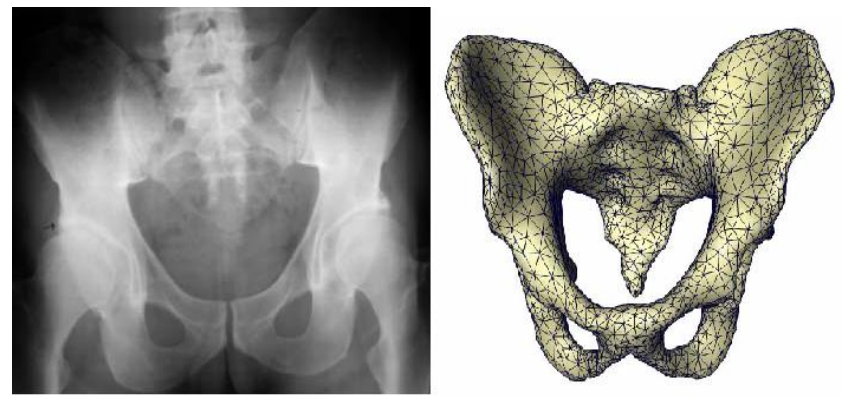

Fig. 2. Reconstruction using Atlas based method

Karade et.al [10] in their paper proposed an algorithm, which is based on the principle of using Kohonen self-organizing maps for correspondence of 2D-3D between the X-ray images inputted and the template image. They used Laplacian surface deformation for final deformation of the template. The 2D- 3D correspondence determined using SOM gives the anchor points for the Laplacian mesh deformation. During deformation, the template mesh does not get distorted and the differential coordinates are preserved due to differential constraints in the Laplacian method. Since the algorithm uses a template model and outer contour from the X-ray image, it can be applied to any part of the skeleton.

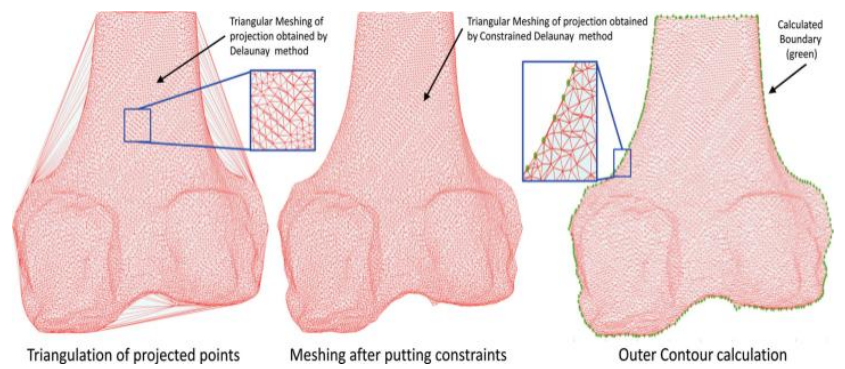

Fig. 3. Detection of boundary points and edges of projection of the 3D point cloud.

Haithem et.al [11] proposed a novel approach for estimating the pose of femur from bi-planar X-ray images. The representation of femur is done using a triangulated surface topology where statistics are built from a sub-space that is linear. The framework of this method is accomplished in forth with the specific projection geometry of the EOS 
system so that an objective function aiming at separation of the bone population from the background can be defined, while primarily deformed towards from bone edges. Future work as mentioned by the authors involves the analyzing of a larger dataset of models and integration of the representation of the prior model. They aim to achieve it using models graphically and using global pose-free parameters prior to model and objective function.

Steffen et.al [12] presented a hierarchical strategy to precisely reconstruct the shape of the acetabulum. The basic concept relies on the registration of two different SSMs. The first SSM is constructed from CT segmented surface models of the hemi-pelvis. The second SSM is constructed on the basis of the hemi pelvis SSM. The mean hemi-pelvis model is used to interactively define the acetabulum region. Subsequently, PCA is applied, resulting in an acetabular patch-SSM. The remaining steps of the hierarchical registration are performed automatically.

Cong et.al [13] lists the different available 3D reconstruction techniques for the available data set. However, they mention that the core principle guiding the $3 \mathrm{D}$ reconstruction process composes of steps that include reading of 2D data slices and arranging them exactly with the real spatial positions, which results in a volumetric data. This volumetric data is further saved into any kind of computer memory. Further, rendering techniques are applied for visualizing the data volume as 3D image. The generally applied rendering techniques for medical images are multi-planar rendering (MPR), surface rendering (SR) and volume rendering (VR).

Wei et.al [14] proposed a technical approach to solve the reconstruction problem that can be summarized as applying mathematical morphology method for edge detection, defining central points of the femur shaft edge line as a shaft datum line for matching. Aided by mark points in both direction X-ray images, they calculated three coordinates of the shaft datum line. They calculated the same shaft datum line from the template femur model, matched the two shaft datum lines by errors between their coordinates' value, registration of the $3 \mathrm{D}$ template model and shape of femur shaft presented by the edge.

Simant [15] in his paper discusses a possible intra-operative method to use a few number of conventional bi-planar X-ray images for reconstructing multiple $3 \mathrm{D}$ bone surfaces. The identification of each bone's edge contours of the X-ray images are performed automatically. Reconstruction of sparse 3D landmark points of very particular bone is performed automatically by combining the $2 \mathrm{D} X$-ray images. In this work, a novel method for identifying the edge contours of the bone is being presented. This information is then further used for identifying the edge contours from varying subjects of interest from the given volume of interest. The reconstructed optimal landmark vertices are then fitted using a statistical shape model, dense point distribution model (DPDM), for reconstruction of a full surface of each and every bone separately.

Liang et.al [16] proposes a method which relies on vertebral contour matching for estimation of vertebral orientations and locations. The adjustment of the vertebral orientations and locations were done automatically. This was carried out by performing a match between the projections of $3 \mathrm{D}$ primitives and the vertebral edges on the X-ray images. This process uses the concept of generalized Hough transform technique with a deformation tolerant matching strategy. They used graphics processing unit to accelerate reconstruction. The proposed reconstruction is based on contour matching to determine the 3-D vertebral orientations and locations. They extended the GHT with a deformation tolerant strategy to find the best matches of the projected contours of the 3-D primitives with the 2-D vertebral edges on PA and LAT radiographs.
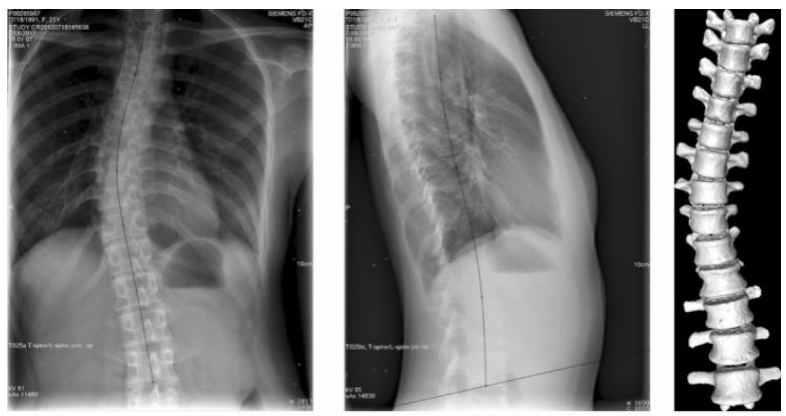

Fig. 4. Reconstruction using Hough transform[16]

Zheng [17] devised an innovative method that requires as input a patient-specific 3D surface model, output from reconstruction of 2D x-ray images as an abettor to produce a patient-specific intensity reconstruction volumetrically using Partial Least Squares Regression (PLSR). No DRR generation is needed. This method consists of two stages. In the first PLSR training stage, using Independent Statistical Shape, Displacement and Appearance (ISSDA) models for dimension reduction, they built two linear mapping functions. In the second $2 \mathrm{D} / 3 \mathrm{D}$ shape and intensity reconstruction stage, they first reconstructed a patientspecific surface model from input $2 \mathrm{D} x$-ray images. They then used those two mapping functions to get a patientspecific displacement field as well as a patient-specific appearance model that is defined in the reference volume.

Zhang et.al [18] in their paper described a method whose core lies behind the principle that the anatomical landmarks can be seen in only two images. It demands as prerequisites in a technical sphere a step for edge detection that results in detection of the 2D contours and a minimum of six calibrating points that will calibrate the bi-planar radiographic environment. This paper chooses the femur as the research object to establish an effective method of 3D reconstruction based on the Direct Linear Transformation algorithm which uses only two orthogonal X-ray images to complete the reconstruction of 3D structure.

Laporte et.al [19] detailed the reconstruction steps to follow the steps as: defining the anatomical regions, identifying the $2 \mathrm{D}$ contours associated to these regions, calculating the $3 \mathrm{D}$ contours and projecting onto the radiographs, associating between points of the X-ray contours and points of the 3D contours after projection, optimizing an initial solution and the optimization of the deformed object so that the distance between the X-ray contours and projected $3 \mathrm{D}$ contours is minimised.

Bras et.al [20] performed 3D reconstruction method using low-dose bi-planar radiographs. An automatic segmentation of grey level and a manual correction of potential segmentation errors were performed to identify all bone voxels in each slice. Finally, the CT scan slices were piled and $3 \mathrm{D}$ femur reconstructions were obtained. Thus, a 3D reconstruction reference was obtained for each proximal femur. The 3D stereo-radiographic reconstruction of the 25 proximal femurs was performed using the NSCC algorithm. This algorithm is based on the deformation of a $3 \mathrm{D}$ generic 
model relatively to be identified using $2 \mathrm{D}$ radiographic contours.

Thomas et.al [21] proposed a method in which statistical atlases are built using point distribution models (PDMs) taking into account a novel hybrid 3D shape model for surface reconstruction. The utility of the hybrid shape model lies in the fact that it abolishes the need of using global scaling to align training examples and generation of instance variables which in a way allows the PDM capture variations in a wider range. The atlases are thus used to reconstruct or register in a deformed manner the surface model of an object using just two to four 2D X-Ray projections of the object.

Murat et.al [22] in their paper presented a computational method that is both cost- and time-effective for generation of a $3 \mathrm{D}$ bone shape using multiple $\mathrm{X}$-ray images. The process starts with a pre-defined 3D template bone shape taken from a clinically normal subject and being scaled to an average size and then followed by scaling and deforming of the template shape. It is continued till an image similar to the input X-Ray image is being produced by the deformed shape outputted by projection on the two-dimensional (2D) plane. The template bone is scaled and deformed using the method of hierarchical freeform deformation. A concatenation of optimization problems is being devised which has the objective of computing the bone's 3D shape. The relevance behind finding this optimization function is to reduce the error calculated between the X-ray image inputted and the projection obtained from the deformed template shape.

Stefano et.al [23] in their paper describes the study and development of a script for a software package to be used commercially for the generation of $3 \mathrm{D}$ models of femurs starting from two orthogonal X-ray images. The script uses the contours of the femur to reconfigure a template model automatically. This template model is needed because a lot of information about the femur shape is missing when only two orthogonal images are used. The FFD method appears to be the best one for the development of a software application for 3D modeling of femurs based both on X-ray images and some template models. The application consists in a script, created using the development toolkit of $3 \mathrm{ds}$ Max.
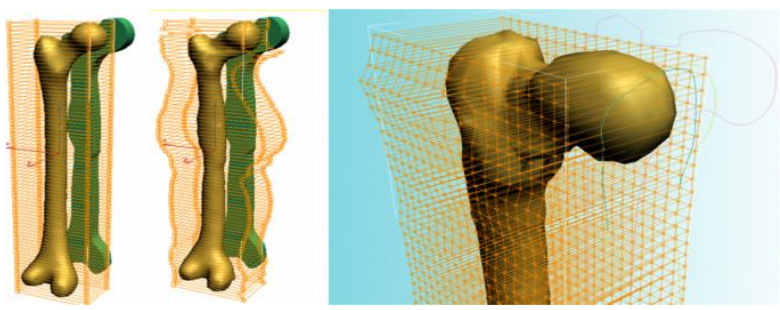

Fig. 5. Screenshot of the reconfiguration process using FFD[23]

Mitton et.al [24] proposed a technique that enables additional landmarks to be reconstructed into $3 \mathrm{D}$, so that they can be identified from only one of the radiographs. The principle behind this method lies in the fact that the deformation of an elastic object respects both stereo-corresponding and nonstereo corresponding observations which are available in varying projections. This core of this technique relies upon the principle that any non-stereo corresponding point will belong to a line that collineates the source of the X-Ray and the projected output of the point being considered from a single view. The core of this method lies in determining the 3D position of these points on their projected line taking into consideration their geometrical and topological constraints.
Kyu Lee et.al [25] in their paper proposed a method for producing the $3 \mathrm{D}$ model of the femoral bone from the conventional bi-planar X-Ray images while associating the anatomical parameters into a referential 3D model. For the $3 \mathrm{D}$ reconstruction to be achieved, the $2 \mathrm{D}$ shape and a definitive number of parameters were firstly measured from $\mathrm{X}$-Ray images. Henceforth, the modification of the referential CT model was performed as: the axial scaling, shearing transformation and radial scaling. Earlier than generating X-Ray based 3D model, the referential 3D femoral model was built by multi-slice CT images. It has a controllable reshaping algorithm based on the anatomical parameters of the patient. The sequential steps followed during the process includes, extracting 2D parameters from X-Ray image, selecting the referential model by CT data, length scaling, anatomical axis transforming, radial scaling, error checking and finally the $3 \mathrm{D}$ reconstruction of the $2 \mathrm{D}$ radiographs.

Zheng et.al [26] in their paper presented a method for building a 2D/3D correspondence which is based upon a non-rigid $2 \mathrm{D}$ point matching process, in which a symmetric nearest neighbor mapping named operator is used along with 2D thin-plate spline based deformations so that a fraction of best matched pairs of $2 \mathrm{D}$ points between features being extracted from the bi-planar X-ray images and the features being extracted from the 3D model can be found. The pairs of points being estimated are further used to build up a set of $3 \mathrm{D}$ pair of points so that a $2 \mathrm{D} / 3 \mathrm{D}$ reconstruction problem turns into a 3D/3D problem, whose solutions are well studied. In this research, a 2D/3D reconstruction scheme that combines a statistical instantiation with a regularized deformation of shape has been produced with the help of the 2D/3D correspondence building method. The proposed 2D/3D correspondence building method belongs to the category of feature-based methods where an explicit extraction of bone edges is required and is different from the intensity-based method.

Kyung et.al [27] in their paper proposed a new reconstruction method for the X-ray of a femur bone. Three CT images were incorporated in this work in order to improve upon the accuracy attained from models constructed in cases of femurs having rotational deformities. A 3D template model of the femur was developed from a healthy person of normal height and weight. Finally, a B-spline free form deformation (FFD) was carried out in order to obtain patient-specific femur models from the 3D template model, using two X-ray images and three CT images of five patients.
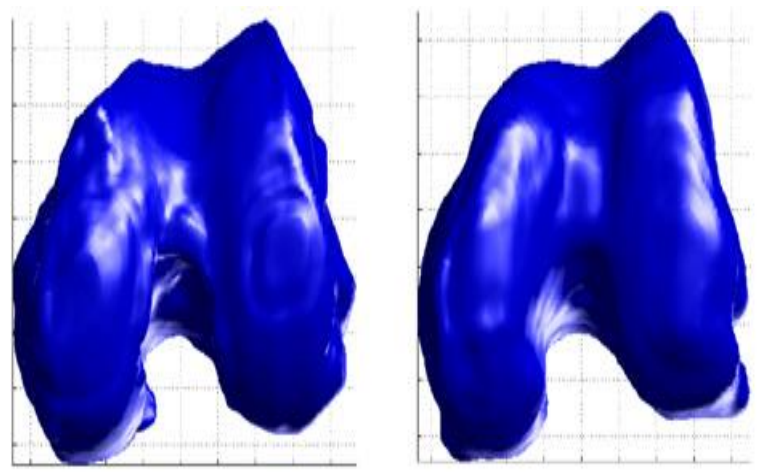

Fig. 6. Angle comparison between 3D models based on CT \& FFD. 


\section{CONCLUSION}

This survey paper reviews the various works carried out in the field of 3D reconstruction of bi-planar X-Ray images. The methods of 3D reconstruction from X-ray images can be classified according to the information used for reconstruction. At one extreme, there are methods in which only the X-ray images are used as the inputs. On the other extreme, there are methods which use a mathematical model containing information of the bone shape as well as its variation. Ehlke et al. [5] included a volume-based statistical shape and intensity models (SSIMs), which result in additional information of volumetric bone density in the reconstructed 3D model. In between the two extremes, there are methods which use the bone shape information in the form of 3D template bone model. The template model is taken as the initial 3D model which is then reconfigured/deformed according to the contour information extracted from the calibrated X-ray images. A method shown by Lee et al.[25] includes scaling, shearing and transformation of different regions of the femur template according to the anatomical parameters measured from X-ray images. In methods presented by Laporte et al.[39], the template bone is elastically deformed using non-stereo corresponding contours (NSCC) algorithm. This method, however, requires manual identification of stereo corresponding points on the calibrated X-rays, which form the constraints for NSCC algorithm-based deformation. In a few other methods, the template bone model is deformed using freeform deformation (FFD) technique where the template is assumed to be enclosed in a bounding box that acts as a control volume. By modifying the control volume parameters, the template inside can be deformed until its projection boundary matches the bone contours extracted from the X-ray images. Gunay et al. [22] have showed a method based on FFD (Hierarchical FFD) where the bounding box is iteratively subdivided into smaller boxes, which can be individually modified to get a shape closest to the desired shape. Another FFD-based method shown by Koh et al. [27] had inclusion of some volumetric information in the form of sparse CT data. Methods based on inputs from $\mathrm{X}$-ray images alone do not give good accuracy since the images have limited information. Methods based on SSM have shown more accurate results but they require a large database. The database can be classified according to age, gender, and ethnicity. The template-based methods using FFD for template reconfiguration have been found to be a viable option for developing software applications for 3 Dreconstruction. However, it has been found that volumebased deformation such as FFD with a few control points is poor in preserving the detailed features of the shape. Hierarchical FFD (for accurate shape matching) requires complex and time-consuming algorithms, and does not consider the topology of the shape under deformation.

\section{REFERENCES}

[1] Cresson T., Branchaud D., Chav R., Godbout B. and de Guise J.A., '3D Shape Reconstruction of bone from two $X$-ray images using 2D/3D non-rigid registration based on moving least-squares deformation', Proceedings of SPIE, Vol. 7623, 76230F, doi: 10.1117/12.844098, Page no. 1-9.

[2] Q. Rizqie, C.Pahl, D.E.O. Dewi, M.A. Ayob, I. Maolana, R. Hermawan, R.D. Soetkino, and E. Suprivanto, '3D Coordinate Reconstruction from 2D Xray images for guided lung biopsy', WSEAS
Transactions on Biology and Biomedicine, EISSN:2224-2902, Vol. 11, 2014, Page no.133-139.

[3] N. Baka, B.L.Kaptein, M.de Bruijne, T. Van Walsum, J.E.Giphart, W.J. Niessen, B.P.F.Lelieveldt, '2D-3D shape reconstruction of the distal femur from stereo XRay imaging using statistical shape models,' Medical Image Analysis 15(2011)840-850, doi:10.1016.

[4] T.Cresson, R.Chav, D. Branchaud, L.Humbert, B.Godbout, B.Aubert, W.Skalli, and J.A.De Guise, 'Coupling 2D/3D registration method and statistical model to perform 3D Reconstruction from partial XRay image data', 31st Annual International Conference of the IEEE EMBS, September 2-6, 2009, pp.10081011 .

[5] Moritz Ehlke, Heiko Ramm, Hans Lamecker, HansChristian Hege, Stefan Zachow, 'Fast generation of virtual X-ray images for reconstruction of 3D anatomy, $1077-2626 / 13$

[6] P.E.Galibarov, P.J.Prendergast, A.B.Lennon, 'A method to reconstruct patient-specific proximal femur surface models from planar pre-operative radiographs', Medical Engineering and Physics 32(2010)1180-1188, doi:10.1016.

[7] P.Gamage, S.Q.Xie, P.Delmas, P.Xu, '3D reconstruction of patient-specific bone models from $2 \mathrm{D}$ radiographs for image guided orthopedic surgery', Digital Image Computing: Techniques and Applications, IEEE, doi: 10.1109, pp.212-216

[8] Oliver Goretzki, '3D Reconstruction of medical images using Java3D', Bachelor Thesis, Luxembourg, 2008-0316.

[9] Hans Lamecker, Thomas H. Wenckebach, HansChristian Hege, 'Atlas-based 3D shape reconstruction from X-ray images'.

[10] Vikas Karade, Bhallamudi Ravi, '3D femur model reconstruction from bi-plane X-Ray images: a novel method based on Laplacian surface deformation', International journal of CARS, doi: 10.1007/s 11548014-1097-6

[11] Haithem Boussaid, Samuel Kadourg, Iasonas Kokkinos, Jean-Yves Lazennec, Guoyan Zheng, Nikos Paragios, '3D model-based reconstruction of the proximal femur from low-dose biplanar X-Ray images', BMVC 2011 http://dx.doi.org/10.5244/c.25.35.

[12] Steffan Schumann, Li Liu, Moritz Tannast, Mathias, Bergmann, Lutz-P. Nolte, Guoyan Zheng, 'An integrated system for 3D hip-joint reconstruction from 2D X-rays: A preliminary validation study', Annuals of Biomedical Engineering, vol.41, No.10, October 2013, pp.2077-2087, doi: 10.1007/s10439-013-0822-6.

[13] Vu Cong, Huynh, Quang Linh, '3D medical image reconstruction', Biomedical Engineering Department, Faculty of Applied Science, HCMC University of Technology.

[14] Wei Wei, Wang Guorong, Chen Hua, '3D reconstruction of a femur shaft using a model and two 2D X-ray images, Proceedings of 2009 4th International Conference on Computer Science and Education, IEEE. 
[15] Simmant Prakoonwit, 'Towards multiple 3D bonesurface identification and reconstruction using few 2D X-ray images for intra-operative applications', Department of computer science and technology,University of Bedfordshire, UK.

[16] JunHua Zhang, Liang Lv, Xingling Shi, Yuanyuan Wang, Fei Guo, Yufeng Zhang, Hongjian Li, '3D Reconstruction of the spine from biplanar radiography based on contour matching using the Hough transform, IEEE Transactions on Biomedical Engineering, Vol.60, No.7, July 2013.

[17] Guoyan Zhang, '3D Volumetric intensity reconstruction from 2D X-ray images using partial least-squares regression', 2013 IEEE 10th International symposium on Biomedical Imaging, pp.1268-1271.

[18] Bin Zhang, Shaobin Sun, Jinwei Sun, Zhiyong Chi, Chunyang $\mathrm{Xi}$, '3D Reconstruction method from biplanar radiography using DLT Algorithm: Application to the femur', 2010 First International Conference on pervasive computing, Signal processing and applications, IEEE, doi:10.1109/PCSPA.2010.68 pp.251-254.

[19] S.Laporte, W.Skalli, J.A.De Guisse, F.Lavaste and D.Mitton(2003): A Bi-planar reconstruction method based on 2D and 3D contours: Application to the distal femur, Computer Methods in Biomechanics and Biomedical Engineering, 6:1, 1-6.

[20] A. Le Bras, S.Laporte, V.Bousson, D.Mitton, J.A. De Guise, J.D.Laredo, W.Skalli, 'Personalised 3DReconstruction of proximal femur from low-dose digital bi-planar radiographs, International Congress Series 1256(2003)214-219, doi: $10.1016 /$ S05315131(03)00285-1.

[21] Thomas S.Y.Tang, Randy E.Ellis, '2D/3D Deformable registration using a hybrid atlas', MICCAI 2005, LNCS 3750, pp.223-230, 2005.

[22] Murat Gunay, Mun-Bo Shim, Kenji Shimada, 'Costand time-effective three-dimensional bone-shape reconstruction from X-Ray images', The International Journal of Medical Robotics and Computer Assisted Surgery 2007; 3:323-335. doi:10.1002/rcs.162.

[23] Stefano Filippi, Barbara Motyl, Camillo Bandera, 'Analysis of existing methods for 3D modeling of femurs starting from two orthogonal images and development of a script for a commercial software package', Computer Methods and programs in Biomedicine 89(2008) 76-82, doi:10.1016/j.cmpb.2007.10.011.

[24] D.Mitton, C.Landry, S.Veron, W.Skalli, F. Lavaste, J.A.De Guise, '3D Reconstruction method from biplanar radiography using non-stereo corresponding points and elastic deformable meshes, Medical and Biological Engineering and Computing.2007, vol.38,pp.133-139.

[25] Moon Kyu Lee, Sang Hyuk Lee, Aram Kim, Inchan Youn, Tae Soo Lee, Nahmkeon Hur, Kuiwon Choi,' The study of femoral 3D reconstruction process based on anatomical parameters using a numerical method', Journal of Biomechanical Science and Engineering, vol.3, no.3, 2008, doi: 10.1299, pp.443-451.
[26] Guoyan Zheng, Sebastian Gollmer, Steffen Schumann, Xiao Dong, Thomas Feilkas, Miguel A.Gonzalez Ballester, 'A 2D/3D correspondence building method for reconstruction of a patient-specific 3D bone surface model using point distribution models and calibrated $\mathrm{X}$ Ray images', Medical Image Analysis 13(2009) 883899, doi:10.1016/j.media.2008.12.003.

[27] Kyung Koh, Yoon Hyuk Kim, Kyungsoo Kim, Won Man Park,' Reconstruction of patient-specific femurs using X-ray and sparse CT Images', Computers in Biology and Medicine 41(2011)421-426, doi:10.1016/j.compbiomed.2011.03.016.

[28] Otomaru I, Nakamoto M, Kagiyama Y, Takao M, Sugano N, Tomiyama N, Tada Y, Sato Y (2012) Automated preoperative planning of femoral stem in total hip arthroplasty from 3D CT data: atlas-based approach and comparative study. Med Image Anal 16(2):415-426.

[29] Ellis RE, Tso CY, Rudan JF, HarrisonMM(1999) A surgical planning and guidance system for high tibial osteotomy. J Comput Aided Surg 4(5):264-274.

[30] Bredbenner TL, Eliason TD, Potter RS, Mason RL, Havill LM, Nicolella DP (2010) Statistical shape modeling describes variation in tibia and femur surface geometry between Control and Incidence groups from the osteoarthritis initiative database. J Biomech 43(9):1780-1786.

[31] Kohonen $\mathrm{T}$ (1982) Self-organised formation of topologically correct feature maps. Biol Cybern 43:5969.

[32] Caponetti L, Fanelli AM (1990) 3D Bone reconstruction from two X-Ray views. In: Proceedings of twelfth annual international conference of the IEEE engineering in medicine and biology society (EMBS 1990); 1990 Nov 1-4; Philadelphia, PA, USA, pp 208-210.

[33] Livyatan, H., Yaniv, Z., Joskowicz, L., 2003. Gradientbased 2D/3D rigid registration of fluoroscopic $\mathrm{x}$-ray to ct. IEEE Transactions on Medical Imaging 22, 13951406.

[34] Fuente M, Schkommodau E, Lutz P,Neuss M,WirtzDC, Radermacher K (2005) 3D reconstruction and navigated removal of femoral bone cement in revision THR based on few fluoroscopic images. In: Proceedings of computer assisted radiology and surgery (CARS 2004); 2005 June 23-26; Chicago, USA, pp 626-631.

[35] Y H Kim, J K Kim, C Choi. Three-dimensional reconstruction of human femur using consecutive computer tomography images and simulated implantation system. J.Med.Eng.Technol, 2004(28): 205-210.

[36] W E Lorensen, H E Cline. Marching cubes: a high resolution 3D surface construction algorithm. Comput Graph, 1987(21): 163-169.

[37] L Caponetti, A M Fanelli. 3D Bone reconstruction from two X-Ray views. Proceedings of Twelfth Annual International Conference of the IEEE Engineering in Medicine and Biology Society, 1990: 208-210.

[38] T.A. Nagelhus Hernes, F. Lindseth, T. Selbekk, A. Wollf, O. Vegard Solberg, E. Harg, O.M. Rygh, G Arne Tangen, I. Rasmussen, S. Augdal, F. Couweleers, 
G. Unsgaard, Computer-assisted 3D ultrasound-guided neurosurgery: technological contributions, including multimodal registration and advanced display, demonstrating future perspectives, Int. J. Med. Robotics Comput. Assist. Surg. 2 (2006) 45-59.

[39] A. Mitulescu, S. Laporte, C. Boulay, J.A. De Guise and W. Skalli, (2000) "3D reconstruction of the pelvis using NSCP technique" in the Meeting of the International Research Society in Spinal Deformities, ClermontFerrand, France, 26-30 May.

[40] S. Coquillart, Extended free-form deformation: a sculpturing tool for 3D geometric modeling, Comput. Graph. 24 (1990) 187-196.

[41] http://www.mathworks.com, accessed on November 18, 2015.
[42] J.W. Fernandez, P. Mithraratne, S.F. Thrupp, M.H Tawhai, P.J. Hunter, Anatomically based geometric modelling of the musculo-skeletal system and other organs, Biomech. Model Mechanobiol. 2 (2004) 139155.

[43] J. Yao. A Statistical Bone Density Atlas and Deformable Medical Image Registration. PhD thesis, Johns Hopkins University, 2001.

[44] S. Benameur, M. Mignotte, S. Parent, H. Labelle, W. Skalli, and J. E. Guise. 3D/2D registration and segmentation of scoliotic vertebrae using statistical models. Computerized Medical Imaging and Graphics, 27(5):321-337, 2003 